\title{
Stabilization of Delay Systems with Nonlinearly Correlated Perturbations by a Constant Controller
}

\author{
G. Fernández-Anaya* and J.J. Flores-Godoy
}

Universidad Iberoamericana, Departamento de Física y Matemáticas, Paseo de la Reforma \#880, Lomas de Santa Fe, México, D. F. 01219, México

\begin{abstract}
In this paper we prove that the same design method of constant controllers for a delay systems given in the literature can be used to stabilize a minimum phase systems with nonlinearly correlated perturbations in the coefficients. This type of perturbation is induced by the composition with strictly positive real functions. This result can also be interpreted on the context of simultaneous and robust stabilization for single-input single-output, linear time invariant delay systems.
\end{abstract}

Keywords: Delay systems, robust stabilizability, simultaneous stabilizability, SPR functions, constant controllers.

\section{INTRODUCTION}

There are just a few results about simultaneous and robust stabilization of delay systems. For example, in [1] and [2] the authors establish delay-dependent results for robust stability and robust stabilization of uncertain linear systems with a time-varying state delay subject to norm-bounded uncertainty via memoryless state feedback. In [3-5], the authors present results of robust stability and robust stabilization, for uncertain linear systems with a timevarying state delay for which these results are independent of the size of the time-delay.

Some solutions to the problem of finding an algorithm to obtain a controller that simultaneously stabilizes $m$ singleinput single-output (SISO) linear time invariant (LTI) systems for the case of a constant controller using the state space description of the system have been given by [6] among others. However, the problem of simultaneous stabilization of delay systems remains without being studied. In [7] and [9], using a frequency domain approach it is consider the question of the existence of a rational controller, for the class of systems composed of a delay element $e^{-h s}$ with (bounded and unbounded) uncertainty in $h$, followed by a plant characterized by a rational transfer function. There are given necessary and sufficient conditions for the existence of such controllers and a controller a design method is described. This controller design yields the entire set of all constant controllers which robustly stabilize a family of systems with uncertainty in the time-delay. Their results are independent of the size of the time-delay.

*Address correspondence to this author at the Universidad Iberoamericana, Departamento de Física y Matemáticas, Paseo de la Reforma \#880, Lomas de Santa Fe, México, D. F. 01219, México;

E-mail: guillermo.fernandez@uia.mx
Nevertheless, the problem of simultaneous and robust stabilization of delay systems with nonlinearly correlated perturbations in the coefficients remains an open problem. It is clear that this last problem is difficult and far from being solved. We will study the simple case when the controller is constant, and the delay systems belong to a particular class.

In this note we prove that the same design method of constant controllers given in [7], can be used with minimumphase delay systems where their coefficients are nonlinearly correlated perturbations induced by the composition with strictly positive real (SPR) functions. Based on this fact, we present a result on simultaneous and robust stabilization for SISO LTI delay systems under nonlinearly correlated perturbations in their coefficients induced by the composition with SPR functions.

\section{PRELIMINARIES}

This section presents definitions and results which will be used throughout the paper.

Notation: Let $R$ and $C$ represent the field of real and complex numbers, respectively. Let $z \in \mathrm{C}$ such that $z=\sigma+j \omega$ with $\sigma \in \mathrm{R}, \omega \in \mathrm{R}$ and $j=\sqrt{-1}$. Also, let denote the real and imaginary part of the complex number $z$ by $\operatorname{Re}[z]=\sigma, \operatorname{Im}[z]=\omega$. Consider the following sets

$$
\begin{aligned}
& \mathrm{C}^{+} \equiv\{z \in \mathrm{C}: \operatorname{Re}[z]>0\} \\
& \operatorname{ImC} \equiv\{z \in \mathrm{C}: \operatorname{Re}[z]=0\}, \\
& \overline{\mathrm{C}}_{e}^{+} \equiv \mathrm{C}^{+} \cup\{\infty\} \cup \operatorname{ImC} \\
& \overline{\mathrm{C}}^{+} \equiv \mathrm{C}^{+} \cup \operatorname{ImC}, \\
& \mathrm{C}_{e}^{+} \equiv \mathrm{C}^{+} \cup\{\infty\} .
\end{aligned}
$$

Definition 1 ([8]) A rational real function $p(s)$ of zero relative degreeis SPR (SPR0 function) if 
i) $\quad p(s)$ is analytic in $\operatorname{Re}[s] \geq 0$,

ii) $\quad \operatorname{Re}[p(j \omega)]>0$ for all $\omega \in \mathrm{R}$.

Now, we present the design method of constant controllers given in [7]. Let a delay system $\bar{p}(s)$ be represented as

$\bar{p}(s)=p(s) e^{-h s}=\frac{N_{p}(s)}{D_{p}(s)} e^{-h s}$

where $N_{p}(s)$ and $D_{p}(s)$ are polynomials as follows:

$N_{p}(s)=n_{r} s^{r}+\cdots+n_{0}$

$D_{p}(s)=d_{m} s^{m}+\cdots+d_{0}$

with $r \leq m$, we assume $N_{p}(s), D_{p}(s)$ are coprime polynomials. Let a rational controller $C(s)$ be given by

$C(s)=\frac{N_{c}(s)}{D_{c}(s)}$

where $N_{c}(s), D_{c}(s)$ are coprime polynomials. Furthermore, the delay system $\bar{p}(s)$ and controller $C(s)$ are connected by a standard unity feedback as shown in Fig. (1).

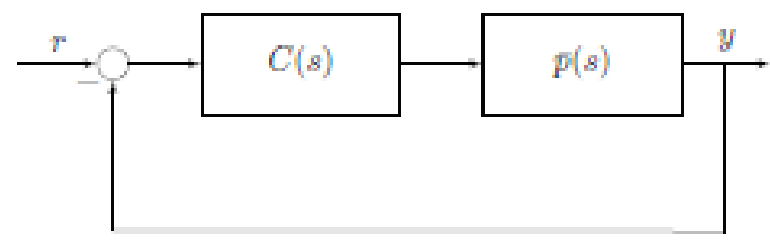

Fig. (1). Unity feedback. $r$ : reference signal, $y$ : system output, $C(s)$ : controller, $\bar{p}(s)$ : system.

The closed-loop system is given by

$$
H(s)=\frac{N_{c}(s) N_{p}(s) e^{-h s}}{D_{c}(s) D_{p}(s)+N_{c}(s) N_{p}(s) e^{-h s}} .
$$

and it is Hurwitz stable if

$$
D_{c}(s) D_{p}(s)+N_{c}(s) N_{p}(s) e^{-h s} \neq 0
$$

for $\operatorname{Re}[s] \geq 0$ for all $h \in[0, \infty)$.

A delay system is stabilized by a constant controller $C(s)=C_{1}$, if the following two conditions are satisfied:

$$
D_{p}(s)+C_{1} N_{p}(s) \neq 0 \text { in } \operatorname{Re}[s] \geq 0 .
$$

$$
\left|C_{1}\right|<\min _{\omega \in \mathrm{R}}\left|p^{-1}(j \omega)\right|
$$

In this note we want to find a constant controller $C_{1}$ such that the closed-loop system
$\mathrm{H}(s)=\frac{C_{1} p(q(s)) e^{-h s}}{1+C_{1} p(q(s)) e^{-h s}}=\frac{C_{1} \frac{N_{p}(q(s))}{D_{p}(q(s))} e^{-h s}}{1+C_{1} \frac{N_{p}(q(s))}{D_{p}(q(s))} e^{-h s}}$

is stable for each SPR0 function $q(s)$. This problem is equivalent to to find a constant controller $C_{1}$ such that

$D_{q}^{n m}(s)\left[D_{p}(q(s))+C_{1} N_{p}(q(s)) e^{-h s}\right] \neq 0$

for $\operatorname{Re}[s] \geq 0$ for all $h \in[0, \infty)$ for each SPR0 function $q(s)$ of order $n$, where $q(s)=\frac{N_{q}(s)}{D_{q}(s)}$.

\section{THE MAIN RESULT}

The main result is given in this section.

Theorem 2 Let $p(s)=\frac{N_{p}(s)}{D_{p}(s)}$ be a stable and minimumphase rational function where $N_{p}(s), D_{p}(s)$ are coprime polynomials. We assume the following conditions:

H1) $\quad q(s)=\frac{N_{q}(s)}{D_{q}(s)}$ is any SPR0 function.

H2) The following inequalities are satisfied:

$$
D_{p}(s)+C_{1} N_{p}(s) \neq 0 \text { in } \operatorname{Re}[s] \geq 0 .
$$

$$
\left|C_{1}\right|<\min _{\omega \in \mathrm{R}}\left|p^{-1}(j \omega)\right| \text {. }
$$

Then:

i)

There exists a constant controller $C_{1}$, such that, it robustly stabilizes $p(q(s)) e^{-h s}$ for all $h \in[0, \infty)$, by continuously varying the coefficients of $q(s)$ in the set

$$
G=\left\{\begin{array}{l|l}
q(s) & \begin{array}{l}
q(s)=\frac{a_{n} s^{n}+\cdots+a_{0}}{b_{n} s^{n}+\cdots+b_{0}} \\
\text { is SPR0 function for } \\
\left(a_{0}, \ldots, a_{n}, b_{0}, \ldots, b_{n}\right) \in \mathrm{E}, \\
\mathrm{E}=Q_{0} \times \cdots \times Q_{n} \times T_{0} \times \cdots \times T_{n}
\end{array}
\end{array}\right\}
$$

where $Q_{i}, T_{i}$ are closed intervals in $\mathrm{R}$ for $i=0, \ldots, n$, i.e.,

$$
D_{p}(q(s))+C_{1} N_{p}(q(s)) e^{-h s} \neq 0
$$

in $\operatorname{Re}[s] \geq 0$ for all $h \in[0, \infty)$ where $q(s) \in G$.

ii) $\quad C_{1}$ simultaneously stabilizes

$p\left(q_{1}(s)\right) e^{-h s}, \ldots, p\left(q_{m}(s)\right) e^{-h s}$ for all $h \in[0, \infty)$, if the orders of $q_{1}(s), \ldots, q_{m}(s)$ are different, where 
$D_{p}\left(q_{j}(s)\right)+C_{1} N_{p}\left(q_{j}(s)\right) e^{-h s} \neq 0$

in $\operatorname{Re}[s] \geq 0$ for all $h \in[0, \infty)$ and for $j=1, \ldots, m$ with $q_{j}(s) \in G$.

Proof. i) Observe that, since the denominator of the rational function $p(s)$ is Hurwitz stable, by Lemma 2 in [7], there exists a rational controller $C(s)=\frac{N_{c}(s)}{D_{c}(s)}$ such that

$D_{c}(s) D_{p}(s)+N_{c}(s) N_{p}(s) e^{-h s} \neq 0$

in $\operatorname{Re}[s] \geq 0$ for all $h \in[0, \infty)$. Now, by Lemma 3 in [7], there also exists a constant robustly stabilizing controller $C_{0}$, such that

$$
D_{p}(s)+C_{0} N_{p}(s) e^{-h s} \neq 0
$$

in $\operatorname{Re}[s] \geq 0$ for all $h \in[0, \infty)$. Note that the constant controller $C_{0}$ is not unique. On the other hand, in [7], the authors establish that the constant controller $C_{1}$, stabilizes the delay system $p(s) e^{-h s}$ for all $h \in[0, \infty)$, i.e.,

$D_{p}(s)+C_{1} N_{p}(s) e^{-h s} \neq 0$

in $\operatorname{Re}[s] \geq 0$ for all $h \in[0, \infty)$, if conditions (C1) and (C2) are satisfied.

Then we need to verify that conditions (a) and (b) are satisfied by the rational function $p(q(s))$ for some constant controller $C_{1}$.

For condition (a): If the function $q(s)$ is a SPR0 function. Then the substitution of the variable $s$ by the function $q(s)$ is an endomorphism over the Euclidian domain $\mathrm{RH}^{\infty}$, and these homomorphisms preserve sums, products, constants and Bezout's identities. Now from the fact that the SPR0 function $q(s)$ is analytic in $\operatorname{Re}[s] \geq 0$ (see Definition 1) and by Theorem 9.9 in [10], we have that

$\operatorname{Re}[q(s)]>0$ for $\operatorname{Re}[s] \geq 0$.

Then the condition (a), for the rational function $p(q(s))$ is satisfied, i.e.,

$D_{p}(q(s))+C_{1} N_{p}(q(s)) \neq 0 \quad$ in $\quad \operatorname{Re}[s] \geq 0$.

As a consequence, if $N_{p}(s)=n_{r} s^{r}+\cdots+n_{0}$, $D_{p}(s)=d_{m} s^{m}+\cdots+d_{0}, \quad r \leq m$ and substituting $q(s)$ by $\frac{N_{q}(s)}{D_{q}(s)}$ in $D_{p}(s)+C_{1} N_{p}(s) \neq 0 \operatorname{inRe}[s] \geq 0$.

Since $N_{q}(s)$ and $D_{q}(s)$ are Hurwitz stable polynomials and by [11], then $D_{q}^{n m}(s) D_{p}(q(s)) \neq 0 \quad$ and $D_{q}^{n m}(s) N_{p}(q(s)) \neq 0$ in $\operatorname{Re}[s] \geq 0$. Note also, that the homomorphism substitution preserves identities and also the zero element. Therefore the homomorphism substitution preserves the last inequality.

For condition (b): By Definition 2.1, condition 1, and since $q(s)$ is SPR0 function, then $q(\operatorname{ImC}) \subseteq \mathrm{C}^{+}$, $q(\operatorname{ImC}) \cap \operatorname{ImC}=\varnothing$ and $q(\operatorname{ImC})$ is a closed bounded domain. The function $p^{-1}(z)$ is analytic for any $z \in \mathrm{C}^{+}$by our hypothesis about $p(s)$ being a minimum phase rational function. Since $p(s)$ is a stable and minimum-phase rational function, $p^{-1}(z) \neq 0$ for any $z \in \mathrm{C}^{+}$. Then, by the Minimum Module Theorem in [12], the minimum

$\min \left|p^{-1}(z)\right|$

for $z \in q(\operatorname{ImC})$ is reached for some $z \in q(\operatorname{ImC})$, since $q(\operatorname{ImC})$ is a closed bounded domain. Now, by the Minimum Module Theorem, the minimum

$\min \left|p^{-1}(z)\right|$

with $z \in \mathrm{C}^{+} \cup \operatorname{ImC}$ is reached for some $z \in \operatorname{ImC}$. But, by Theorem 6 in [12],

$\left|p^{-1}(z)\right|>\min _{z \in \mathrm{C}^{+} \cup \operatorname{ImC}}\left|p^{-1}(z)\right|$

for every $z \in \mathrm{C}^{+}$. As a consequence, from the facts that $q(\operatorname{ImC}) \cap \operatorname{ImC}=\varnothing, q(\operatorname{ImC}) \subseteq \mathrm{C}^{+}$, Theorem 6 [12] and the Minimum Module Theorem

$\min _{z \in \mathrm{C}^{+} \cup \mathrm{ImC}}\left|p^{-1}(z)\right|=\min _{\omega \in \mathrm{R}}\left|p^{-1}(j \omega)\right|<\min _{z \in q(\mathrm{ImC})}\left|p^{-1}(z)\right|$.

Using the condition (b), we have that

$\left|C_{1}\right|<\min _{\omega \in \mathrm{R}}\left|p^{-1}(j \omega)\right|<\min _{z \in q(\operatorname{ImC})}\left|p^{-1}(z)\right|$.

Since

$\min _{z \in q(\operatorname{ImC})}\left|p^{-1}(z)\right| \leq \min _{\omega \in \mathrm{R}}\left|p^{-1}(q(j \omega))\right|$

because $z \in q(\operatorname{ImC})$ is free, while $z \in\{z=q(j \omega) \mid \omega \in \mathrm{R}\}$ is restricted to the range of the complex function $q(j \omega)$ for each value $j \omega \in \operatorname{ImC}$. Then condition (b) is satisfied for the rational function $p(q(s))$ i.e.,

$\left|C_{1}\right|<\min _{\omega \in \mathrm{R}}\left|p^{-1}(q(j \omega))\right|$.

Then, the two conditions (a) and (b) are satisfied. Now, since $q(s) \in G$, the constant controller $C_{1}$, robustly stabilizes the delay system $p(q(s)) e^{-h s}$ for all $h \in[0, \infty)$.

ii) This proof is similar to the previous one using the same controller $C_{1}$, but now taking the orders of the SPR0 functions $q_{1}(s), \ldots, q_{m}(s)$ different.

The assumption that the system is stable guarantees the existence of a constant controller by Lemma 2 and Lemma 3 in [7]. Note that this result is among the few results that exist 
about simultaneous stabilization and robust stabilization of delay systems using constant controllers with nonlinearly correlated perturbations in the coefficients induced by the composition of SPR0 functions. (see [11] and [13]).

Also, observe that conditions (a) and (b) are sufficient conditions for the constant controller $C_{1}$ to stabilizes the rational function $p(s)$. Therefore, the same sufficient conditions to stabilize the delay system $p(s) e^{-h s}$, with a constant controller $C_{1}$, are also sufficient to stabilize the delay system $p(q(s)) e^{-h s}$, with the same constant controller $C_{1}$, for each SPR0 function $q(s)$ if the rational function $p(s)$ is minimum-phase rational function. Another consequence is that the design method presented in [7] for constant controllers which robustly stabilize the delay system $p(s) e^{-h s}$, it is also valid for the delay systems $p(q(s)) e^{-h s}$ and can be extended to interval plants. Moreover, the unique additional condition for the rational function $p(s)$ is that it must be a minimum-phase rational function. On the other hand, Theorem 2 can be generalized to other contexts for SPR functions (see [14]).

\section{EXAMPLE}

In this section we give an examples to show the applicability of the result.

Example 1. In the example 1 of [7], the constant controllers which stabilizes

$$
\bar{p}(s)=p(s) e^{-h s}=\frac{s+1}{s^{2}+5 s+6} e^{-h s}
$$

for all $h \in[0, \infty)$ are $C_{1} \in(-4.56,4.56)$. Based on this example, we show that these $C_{1}$, simultaneously stabilize

$$
\bar{p}_{1}(s)=p_{1}(s) e^{-h s}=\frac{10 s^{2}+17 s+6}{63 s^{2}+119 s+63} e^{-h s}
$$

and

$$
\begin{aligned}
\bar{p}_{2}(s) & =p_{2}(s) e^{-h s} \\
& =\frac{5\left(2 s^{4}+21 s^{3}+68 s^{2}+69 s+20\right)}{63 s^{4}+653 s^{3}+2127 s^{2}+2255 s+750} e^{-h s}
\end{aligned}
$$

for all $h \in[0, \infty)$, also. Note that $p_{1}(s)=p\left(\frac{3 s+5}{2 s+1}\right)$ and $p_{2}(s)=p\left(\left(\frac{s+3}{s+5}\right)\left(\frac{3 s+5}{2 s+1}\right)\right)$. Also note that $\frac{2 s+3}{5 s+7}$ is an SPR0 function, and $\left(\frac{s+3}{s+5}\right)\left(\frac{3 s+5}{2 s+1}\right)$ is an SPR0 function. Then, by Theorem 2, the constant controllers $C_{1} \in(-4.56,4.56) \quad$ simultaneously stabilize $\quad \bar{p}_{1}(s)$ and $\bar{p}_{2}(s)$ for all $h \in[0, \infty)$. On the other hand, we can verify that

$\left|C_{1}\right|=4.56 \leq \min _{\omega}\left|p_{1}^{-1}(j \omega)\right|=6.34$ and

$$
\left|C_{1}\right|=4.56 \leq \min _{\omega}\left|p_{2}^{-1}(j \omega)\right|=6.11
$$

based on the example 1 in [7] too.

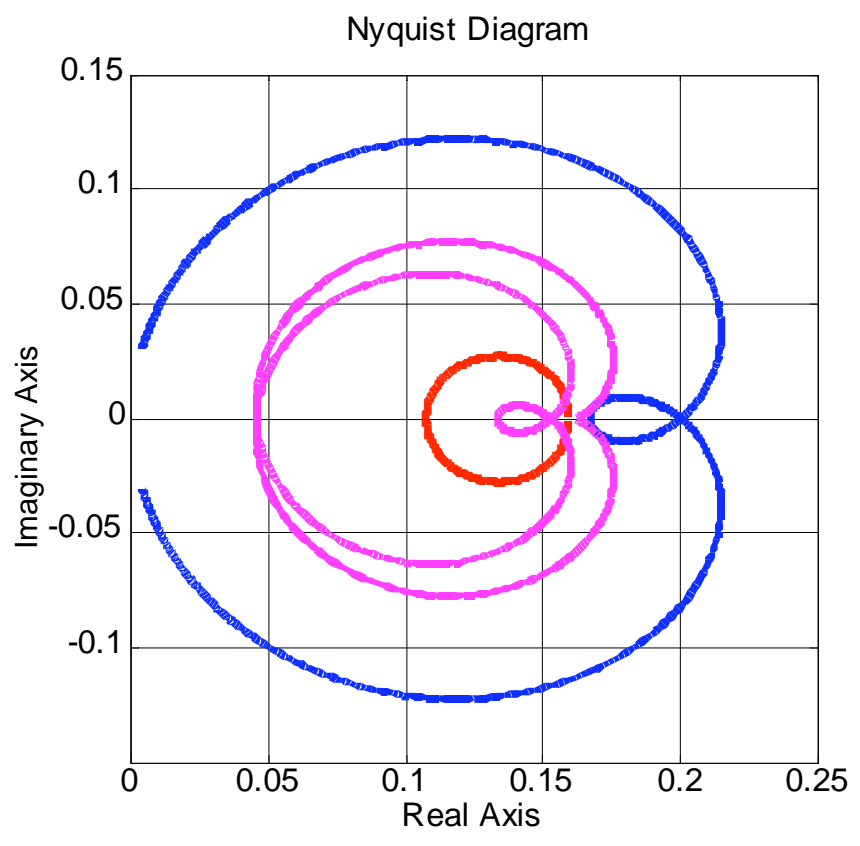

Fig. (2). Nyquist diagram for the Example 1 systems. $\$ p(j l o m e g a) \$:$ solid blue line, \$p_1(jlomega)\$: dashed red line, \$p_2(jlomega)\$: dotted magenta line.

As an observation, the controller design condition, implies that the largest gain needed to cross the point $(1,0)$ on the Nyquist diagram for $\left|p^{-1}(j \omega)\right|$, notice that for $p_{1}$ and $p_{2}$, the composition of $p$ with their respective SPR functions, their Nyquist diagrams are contained inside the Nyquist diagram of $p$, as can it be seen in Figure 2, from here it is possible to visualize why the gain for the controller will stabilize the plants in the example.

In Fig. (3) we see the Nyquist diagrams for the delayed systems for which the stability is ensure by the constant controller $C_{1}$.

\section{CONCLUSIONS}

Based on some results of a delay systems class, we prove that the same design method of constant controllers given in the literature stabilizes delay systems with nonlinearly correlated perturbations in the coefficients induced by the composition with SPR functions, if the plant is minimumphase. Based on this fact, we have given a result on simultaneous and robust stabilization for SISO LTI delay systems, with nonlinearly correlated perturbations in the coefficients, induced by the composition with SPR0 functions, using constant controllers. Moreover, the result works for almost any SPR function. Also is possible to find the entire set of all constant controllers which robustly stabilize of the system, for each SPR0 function, using the technics found in the literature. 


\section{Nyquist Diagram}

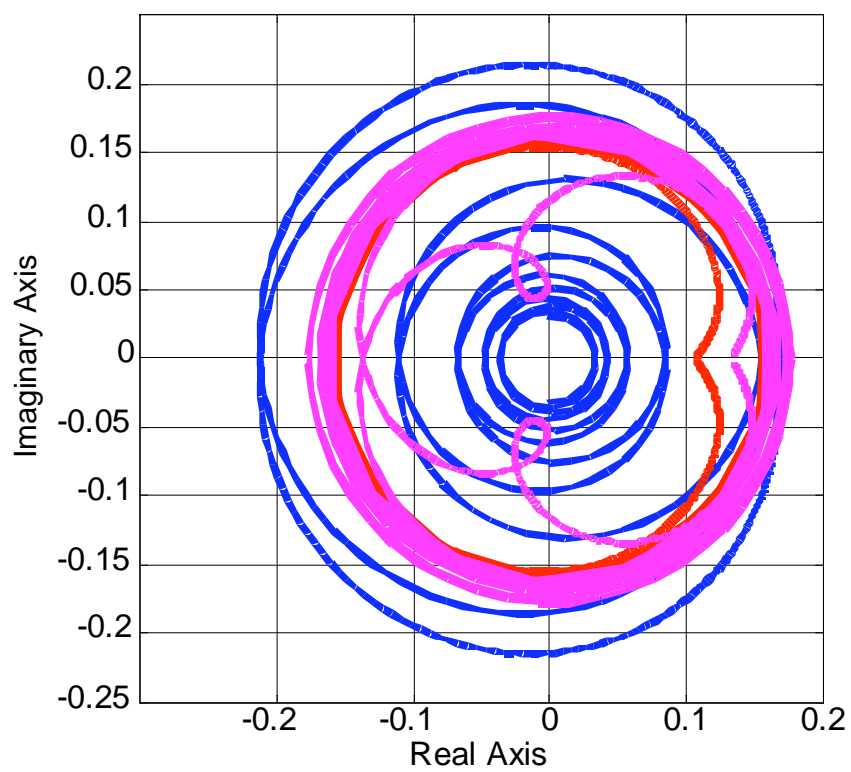

Fig. (3). Nyquist diagram for the Example 1 systems. For $h=1$, $\bar{p}(j \omega)$ : solid blue line, $\bar{p}_{1}(j \omega)$ : dashed red line, $\bar{p}_{2}(j \omega)$ : dotted magenta line.

\section{REFERENCES}

X. Li and C. E. Souza, "Criteria for robust stability and stabilization of uncertain linear systems with state delay" Automatica, vol. 33, no. 9, pp. 1657-1662, September 1997.

[2] S. I. Niculescu, C. E. de Souza, J. M. Dion, and L. Dugard, "Robust stability and stabilization of uncertain linear systems with state delay: Single delay case (I)" In Proc. IFAC Symp. on Robust Control Design, Rio de Janerio, Brazil, September 1994.
[3] Y. Cao and Y. Sun, "Robust stabilization of uncertain systems with time-varying multistate delay" IEEE Trans. Automat. Contr., vol. 43, no. 10, pp. 1484-1488, October 1998.

[4] J. S. Luo, A. Johnson, and P. P. J. van den Bosch, "Delayindependent robust stability of uncertain linear systems" Syst. Contr. Lett., vol. 24, no. 1, pp. 33-39, January 1995.

[5] S. Phoojaruenchanachai and K. Furuta, "Memoryless stabilization of uncertain linear sistems including time-varying state delays" IEEE Trans. Automat. Contr., vol. 37, no. 7, pp. 1022-1026, July 1992.

[6] G. D. Howitt and R. Luus, "Control of a collection of linear systems by linear state feedback control" Int. J. Control, vol. 58 , no. 1, pp. 79-96, July 1993.

[7] L. Naimark, J. Kogan, A. Leizarowitz, and E. Zeheb, "On rational stabilizing controllers for interval delay systems" In L. Dugard and E. I. Verriest, editors, Stability and Control of Time-delay Systems, pages 185-204. Springer-Verlag, London, 1998

[8] P. A. Ioannou and G. Tao, "Frequency domain conditions for strictly positive real functions" IEEE Trans. Automat. Contr., vol. 32, no.1, pp. 53-54, January 1987.

[9] L. Naimark and E. Zeheb, "All constant gain stabilizing controllers for an interval delay system with uncertain parameters" Automatica, vol. 33, no. 9, pp. 1669-1675, September 1997.

[10] S. P. Bhattacharyya, H. Chapellat, and L. H. Keel, Robust Control: The parametric approach. Prentice Hall, 1995.

[11] G. Fernádez-Anaya, "Preservation of SPR functions and stabilization by substitutions in SISO plants" IEEE Trans. Automat. Contr., vol. 44, no. 11, pp. 2171-2173, November 1999.

[12] R. V. Churchill, Complex variables and applications. McGrawHill, second edition, 1960.

[13] L. Wang, "Robust stability of a class of polynomial families under nonlinearly correlated perturbations" Syst. Contr. Lett., vol. 30, no.1, pp. 25-30, March 1997.

[14] G. Fernádez-Anaya, J. C. Marítnez, and V. Kučera, " $H_{\infty}$ robustness properties preservation in siso systems when applying spr substitutions" Int. J. Control, vol. 76, no. 7, pp. 728-740, May 2003.

(C) Deniziak and Tomaszewski; Licensee Bentham Open

This is an open access article distributed under the terms of the Creative Commons Attribution License (http://creativecommons.org/license/by/2.5/), which permits unrestrictive use, distribution, and reproduction in any medium, provided the original work is properly cited. 